Ann. Sci. For., 1985, 42 (4), 397-410

\title{
Optimisation des relations entre transpiration et photosynthèse. Observations à propos des méthodologies d'étude
}

\author{
J.M. GUEHL \\ I.N.R.A., Station de Sylviculture et Production \\ Centre de Recherches forestières de Nancy, Champenoux, F 54280 Seichamps
}

\begin{abstract}
Résumé
Cowan et Farquhar ont proposé en 1977 une théorie d'optimisation des relations entre transpiration et photosynthèse chez les végétaux supérieurs. Au cours d'un intervalle de temps donné, l'optimisation du coût en eau pour la fixation de $\mathrm{CO}_{2}$ nécessite la constance au cours du temps du rapport instantané

$$
\frac{\partial T R / \partial g_{s}}{\partial F_{v} / \partial g_{s}}
$$

où TR est le taux de transpiration, $F_{\mathrm{N}}$ le taux de photosynthèse nette et gis la conductance stomatique pour le $\mathrm{CO}_{2}$. Les études relatives à la théric d'optimisation ont souvent eu pour support expérimental l'étude en laboratoire des variations de $F_{x}$ et TR en réponse à des situations de sécheresse atmosphérique croissante, dans l'hypothèse de variations de $F_{x}$ uniquement liées ì celles de gs.

Il est montré dans la présente étude que cette hypothèse n'est pas vérifiće dans le cas du sapin pectiné. Le recours à la méthodologie classique pour l'étude de l'optimisation est alors impossible. Une méthodologie plus générale est proposée, basée sur une analyse des données permise par leur représentation sous forme de relations entre $F_{x}$ ef la concentration intercellulaire en $\mathrm{CO}_{\text {. }}$.
\end{abstract}

\section{Introduction}

L'assimilation photosynthétique du carbone, substrat essentiel pout la croissance des végétaux, s'accompagne de pertes d'eau par transpiration. La relation entre transpiration et photosynthèse est déterminée par le degré d'ouverture des stomates. Les mouvements stomatiques constituent le moyen le plus rapide pour les végétaux supérieurs de réguler leurs flux d'échanges de matière avec l'atmosphère en réaction aux variations des facteurs de l'environnement. Pour beaucoup de plantes, les stomates s'ouvrent lorsque les conditions sont favorables à la photosynthèse et se ferment lorsque la transpiration devient trop importante (RASchKe, 1975, 1979; Farouiar, 1978). Dans le détail, ce fonctionnement est fortement modulé suivant les espèces ou même 
les provenances à l'intéricur d'une espèce (Aussenac \& Granter, 1978; Aussienac, 1980). La survie d'unc cspèce dans un milieu donné est liée à l'existence d'un fonctionnement stomatique spécifique réalisant un compromis viable entre gains de carbone et pertes d'cau.

Cowan (1977) et Cowan \& Farouhar (1977) ont proposé une théoric d'optimisation de l'utilisation de l'eau par les végétaux supérieurs en relation avec l'assimilation carbonée. Ils se sont attachés à préciser des critères analytiques pouvant permettre d'apprécier le degré de réalisation de cette optimisation pour unc espèce et un intervalle de temps donnés, au cours duquel transpiration et photosynthèse varient.

Après un court exposé de la théorie d'optimisation, nous nous attacherons ici à montrer comment cette théorie peut s'appliquer à l'étude de l'influence d'une sécheresse atmosphérique sur les échanges gazeux du sapin pectiné.

\section{Théorie d'optimisation des relations entre transpiration et assimilation carbonée}


$\mathrm{mg} \mathrm{CO.2} \cdot \mathrm{m}^{-2} \cdot \mathrm{s}^{-1}$ ) à travers les stomates à un instant donné on pour expression :

$$
\begin{gathered}
\mathrm{F}_{x}=\mathrm{g}_{*}\left(\mathrm{c}_{\mathrm{i}}-\mathrm{c}_{\mathrm{i}}\right) \\
\mathrm{TR}=1.6 \mathrm{~g}_{*}\left(\mathrm{c}^{\prime} \mathrm{f}-\mathrm{c}^{\prime} \mathrm{a}\right)=1.6 \mathrm{~g}_{*} \cdot \Delta \mathrm{c}^{\prime}
\end{gathered}
$$

où $\mathrm{g}_{4}\left(\mathrm{~m} \cdot \mathrm{s}^{-1}\right)$ est la conductance stomatique pour le $\mathrm{CO}_{2,}, c_{41}\left(\mathrm{mg} \cdot \mathrm{m}^{-3}\right)$ est la concentration atmosphérique en $\mathrm{CO}_{2,}, \mathrm{c}_{\mathrm{i}}\left(\mathrm{mg} \cdot \mathrm{m}^{-3}\right)$ est la concentration intercellulaire en $\mathrm{CO}_{2}$, $\mathrm{c}^{\prime} f$ et $\mathrm{c}^{\prime} \mathrm{a}\left(\mathrm{mg} \cdot \mathrm{m}^{-3}\right)$ sont respectivement les concentrations en vapeur d'eau aux sites de changement de phase de l'eau dans les feuilles et dans l'atmosphère, 1.6 est un coefficient égal au rapport des coefficients de diffusion de $\mathrm{H}_{2} \mathrm{O}$ et $\mathrm{CO}$., à travers les stomates, $\Delta c^{\prime}$ est égal à la différence $c^{\prime} f-c^{\prime} a$.

Dans les équations (1) et (2) on ne tient pas compte des conductances diffusives liées à la couche limite, nettement supérieures aux conductances stomatiques. De même, on néglige la transpiration cuticulaire par rapport au flux passant par les stomates.

On vérifie que, dans les conditions expérimentales utiliséés dans la présente étude, la température des aiguilles est très voisine de la température de l'ait cécart inférieur' à 0,2 "C). éf peut donc être assimilé à la concentration saturante en vapeur d'cau à la température de l'air.

Une relation supplémentaire peut être définie entre les variables $f_{x}$ el $c_{i}$ de l'équation (1) :

$$
\mathrm{F}_{\mathrm{X}}=\mathrm{D}\left(\mathrm{c}_{\mathrm{i}}\right)
$$

où $D$ est une fonction traduisant l'efficacité de la machineric photosynthétique (Jones, 1973). La fonction D est variable dans le temps, elle dépend de l'état physiologique de la feuille et de l'ensemble des facteurs extérieurs, autres que la concentration en $\mathrm{CO}$., de l'air, influençant les processus non stomatiques de la photo- 
synthèse (température, éclairement, facteurs hydriques...). Malgré les progrès réalisés dans la modélisation de la photosynthèse à l'échelle de la feuille (voir par exemple FAROuHar et al., 1980 b), la fonction D ne peut pas, à l'heure actuelle, être modélisée de façon satisfaisante à partir de données expérimentáles simples. Elle peut être établie expérimentalement en étudiant en laboratoire les variations de $F_{x}$ el TR en fonction de $c_{n}\left(c_{i}\right.$ est déterminé d'après les équations (1) et (2)), toutes choses égales par ailleurs. Nous avons établi de cette façon une telle fonction $D\left(c_{i}\right)$ pour les conditions expérimentales du point 1 de la figure 2. La relation est curviligne et se caractérise par un point de compensation (I) de la photosynthèse pour le $\mathrm{CO}$. égal à $68 \mathrm{mg} \cdot \mathrm{m}^{-: 3}$ (soit $37 \mathrm{ul}^{\mathrm{l}} \cdot \mathrm{I}^{-1}$ ).

Les paramètres $c_{i 1}$, eff et c'a des équations (1) et (2), imposés par le milicu extérieur, sont supposés connus; les équations (1), (2) et (3) permettent alors de définir une fonction $\mathrm{E}$ valable pour un instant $t$ donné :

$$
\mathrm{TR}=\mathrm{E}\left(\mathrm{F}_{\mathrm{N}}\right)
$$

où gs est un paramètre implicite.

L'hypothèse de départ utilisée par Cowan (1977) et Cowan \& Farouhar (1977) est l'optimisation, au cours d'un intervalle de temps $(\mathrm{O}, \mathrm{T})$ durant lequel par exemple les facteurs climatiques varient, du taux moyen d'eau transpirée $\overline{\mathrm{TR}}$ par rapport au taux moyen de carbone assimilé $\bar{F}_{N}$. Le chemin parcouru par $F_{X}$ et $T R$ au cours de cet intervalle de temps est optimal s'il est impossible de concevoir un autre chemin par lequel il puisse être possible d'obtenir une diminution de TR sans entraîner une diminution de $\mathrm{F}_{\mathrm{X}}$. Les auteurs démontrent que cette hypothèse est équivalente, pour des intervalles de temps ne dépassant pas la journée, à :

$$
\left(\frac{\partial T R}{\partial F_{x}}\right)_{t}=\lambda
$$

et

$$
\left(\frac{\partial^{2} \mathrm{TR}}{\partial F_{X^{2}}{ }^{2}}\right)_{t}>0
$$

où $\hat{\lambda}$ est une constante positive dépendant de $F_{N}$, TR et $F_{x}$ varient à l'instant t suivant la fonction de transpiration $\mathrm{E}$.

Pour les plantes à fonctionnement photosynthétique de type $\mathrm{C}_{3}$, l'inégalité (6) est généralement vérifiée, sauf dans de très rares situations (Jonss, 1980). L'équation (5) est plus contraignante, le rapport $\partial \mathrm{TR} / \widehat{\partial} \mathrm{F}_{\mathrm{N}}$ est un coût marginal unitaire en cau pour l'assimilation de $\mathrm{CO}_{2}$; le fonctionnement stomatique optimal est donc celui qui assure la constance de ce coût marginal lorsque $t$ varie (COWAN, 1982).

Il est intéressant d'exprimer $\partial \mathrm{TR} / \partial \mathrm{F}_{\mathrm{X}}$ comme le rapport des sensibilités de la transpiration et de la photosynthèse à la conductance stomatique, en effet :

$$
\frac{\partial T R}{\partial F_{X}}=\frac{\partial T R}{\frac{\partial g_{s}}{\partial g_{s}}}=1.6 \frac{\left(c^{\prime} f-c^{\prime} a\right)}{\frac{\partial F_{N}}{\partial g_{s}}}
$$


Le rapport $\partial \mathrm{F}_{\mathrm{N}} / \partial \mathrm{g}_{\mathrm{s}}$ a été déterminé dans une série de travaux (FArouhar ét al.. 1980 a ; Hall \& Schulze, 1980 ; Meinzer, 1982) en utilisant des déficits de saturation de l'air croissants pour moteur des variations de $F_{x}$ et $g_{*}$ et en adoptant pour valeur de $\partial \mathrm{F}_{N} / \partial \mathrm{g}_{s}$ la pente locale de la relation $\mathrm{F}_{\mathrm{N}}\left(\mathrm{g}_{\mathrm{s}}\right)$ à l'instant $\mathrm{t}$. Ce procédé sera désigné par «méthode graphique directe» dans la suite de cet article. En fait. par définition même de la dérivation partielle, ce procédé n'est acceptable que si les variations de $F_{X}$ sont uniquement liées à celles de $g_{*}$.

Nous avons entrepris de tester cette hypothèse par une étude expérimentale des effets d'une sécheresse atmosphérique croissante sur les échanges gazeux de $\mathrm{CO}$. et $\mathrm{H}_{2} \mathrm{O}$ chez le sapin pectiné.

\section{Etude expérimentale de l'influence de la sécheresse atmosphérique sur la photosynthèse et la conductance stomatique du sapin pectiné}

\subsection{Matériel et méthodes}

Les échanges gazeux de $\mathrm{CO}_{2,}$ et $\mathrm{H}_{2 .} \mathrm{O}$ ont élé mesurés, en août 1984 d'une part, et fin septembre-début octobre d'autre part, sur des rameaux coupés prélevés dans des plantations situćes à proximité du Centre de Recherches Forestières de Nancy dans le Nord-Est de la France (altitude $255 \mathrm{~m}$ ), âgées de 17 ans. La hauteur des arbres était de $5 \mathrm{~m}$. Les provenances étudiées étaient :

- Ecouves, sapin de plaine de Basse-Normandie (altitude $300 \mathrm{~m}$, latitude $48^{\circ} 31^{\prime} \mathrm{N}$, longitude $0^{\circ} 04^{\prime} \mathrm{E}$ ). Climat océanique caractérisé par des pluies fines et fréquentes et une forte nébulosité, mais les risques de sécheresses estivales sont réels.

- Nebras, sapin de moyenne altitude dans l'Aude (altitude $600 \mathrm{~m}$, latitude $42^{\circ} 54^{\prime} \mathrm{N}$, longitude $2^{\circ} 08^{\prime} \mathrm{E}$ ) sur le Piémont pyrénéen. Les influences méditerranéennes se traduisent par un vent d'Est chargé d'humidité, les sécheresses estivales sont généralement bien accusées.

Pour chaque provenance, trois branches provenant de deux arbres différents pour Ecouves et de trois arbres différents pour Nebias étaient prélevées entre 2 et $3 \mathrm{~m}$ de hauteur sur des arbres de bordure et l'extrémité coupée était mise sous eau immédiatement. Au laboratoire, on a recoupé sous eau les pousses terminales de ces branches (portant uniquement des aiguilles de 1984 exposées au plein soleil) et, sans interrompre l'alimentation hydrique des rameaux, on a déterminé $F_{x}$ et $T R$ globalement pour les 3 rameaux à l'aide d'un système de mesure Sirigor-Siemens. Les conditions expérimentales étaient les suivantes : température de l'air égale à 20 "C. éclairement au niveau des rameaux correspondant à une densité de flux dè photons de $460, \mathrm{~mol} \cdot \mathrm{m}^{-2} \cdot \mathrm{s}^{-1}$ dans la bande $400-700 \mathrm{~nm}$, concentration en $\mathrm{CO}$. de l'air égale à $549 \mathrm{mg} \cdot \mathrm{m}^{-3}$, humidité relative de l'air égale à 80 p. 100, 70 p. 100,60 p. 100 , 50 p. 100 , puis 35 p. 100 , soit des écarts $\Lambda c^{\prime}$ variant entre 3470 et $11270 \mathrm{mg} \cdot \mathrm{m}$ ":.

A partir de ces mesures, on a déterminé g. à l'aide de l'équation (2) pour chaque jeu de conditions expérimentales. 


\subsection{Résultats}

On a représenté par la figure 1 les relations entre $g_{s}$ et $F_{X}$ obtenues pour les deux provenances en août 1984 (courbes I) et en septembre-octobre 1984 (courbes II). Seule la courbe obtenue au 28 septembre pour la provenance Ecovves est qualitativement identique aux courbes de concavité négative obtenues par Farouhar et al.
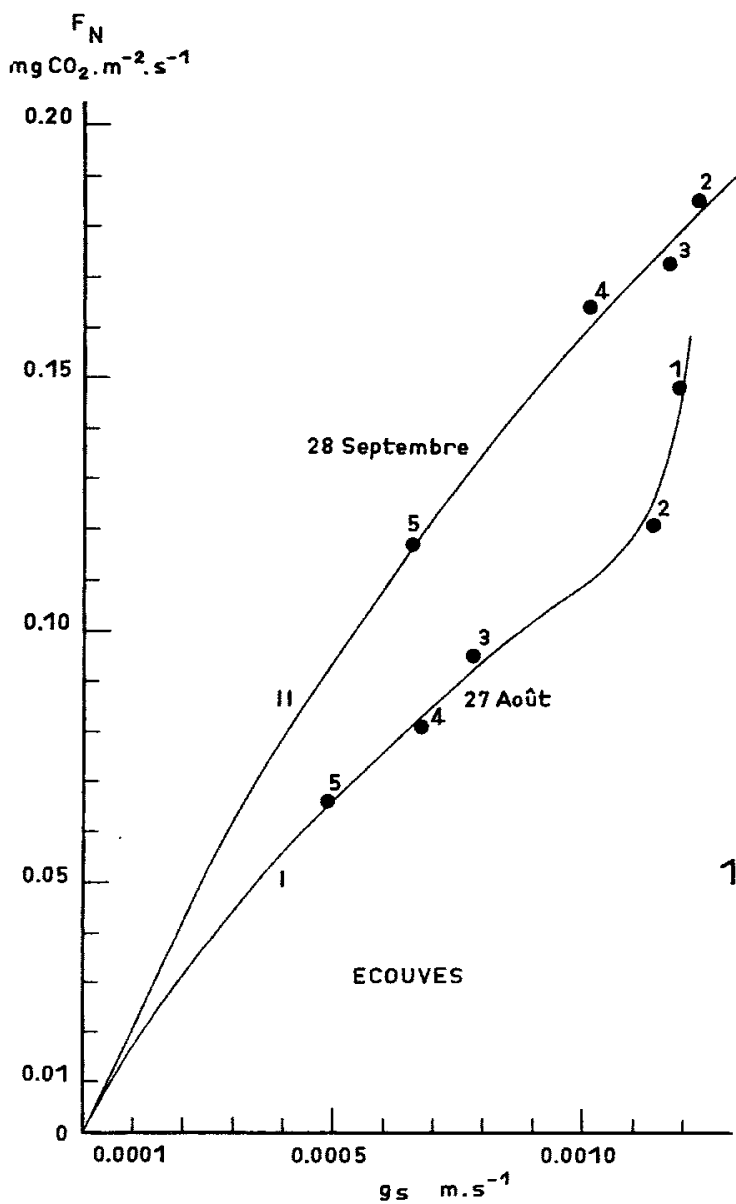

FIG. 1
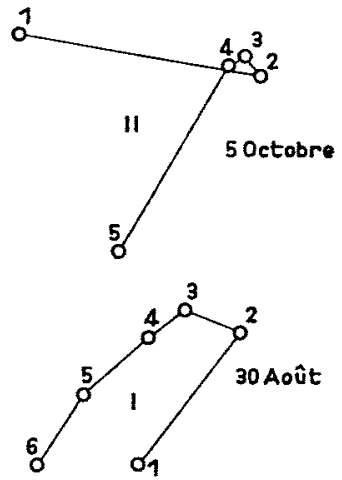

1984

Relations entre l'intensité de photosynthèse nette $\left(F_{S}\right)$ et la conductance stomatique $\left(g_{S}\right)$ pour les provenances Ecouves (O) et Nebias (O) da sapin pectiné obtenues dans les conditions suivantes.

Température de l'air, $20^{\circ} \mathrm{C}$; éclairement, $460 \mu \mathrm{mol} \cdot \mathrm{m}^{-2} \cdot \mathrm{s}^{-1}$; concentration en CO. de l'air, $549 \mathrm{mg} \cdot \mathrm{m}^{-3}$; différences de concentration en vapeur d'eau (mg $\mathrm{H}_{2} \mathrm{O} \cdot \mathrm{m}^{-3}$ ) entre les feuilles et l'atmosphère $\left(\Delta c^{\prime}\right), 3470(1), 5220(2), 6940(3), 8670(4), 11270(5)$, 13005 (6) $\mathrm{mg}-\mathrm{ml}^{-3}$.

Relationships between net $\mathrm{CO}_{2}$ assimilation rate $\left(F_{N}\right)$ and stomatal conductance (g.) for the provenances Ecouves (O) and Nebias (O) of Abies alba.

Air temperature, $20^{\circ} \mathrm{C}$; incident photosynthetic photon flax density, 460 umol $\cdot \mathrm{m}^{-2} \cdot \mathrm{s}^{-1}$ : atmospheric $\mathrm{CO}_{2}$ concentration, $549 \mathrm{mg} \cdot \mathrm{m}^{\cdots ;} ;$ leaf-to-air humidity difference, 3470 (points 1), $5200(2), 6940$ (3), 8670 (4), 11270 (5) and 13005 (6) $\mathrm{mg} \cdot \mathrm{m}^{\cdots}$.". 
(1980 a) sur Nicotinia glatca et Corylus avellana, et par Meinzro (1982) sur Pseudotsuga menziesii et pourrait faire penser à des variations de $F_{Y}$ entièrement dues à celles de gs (Wong el al.. 1979; Bethenod, 1983). Les trois autres courbes ne sont pas compatibles avec cette hypothèse, soit en raison de l'existence d'un point d'inflexion dans la relation (courbe Ecouves 1), soit en raison d'une dispersion de points telle qu'aucune relation fonctionnelle ne puisse être mise en évidence (Nebias). Le recours à la solution graphique directe pour estimer $\partial \mathrm{F}_{N} / \partial \mathrm{g}_{\mathrm{s}}$ est donc impossible ici.

La nature exacte de la relation entre $F_{X}$ et $g_{*}$ peut être déterminće grâce à une méthode proposée par Prioul et al. (1984), (voir aussi Cornic et al., 1983). Cette méthode permet de déterminer les composantes d'origine stomatique et celle d'origine mésophyllienne lors de variations de $\mathrm{F}_{\mathrm{Y}}$.

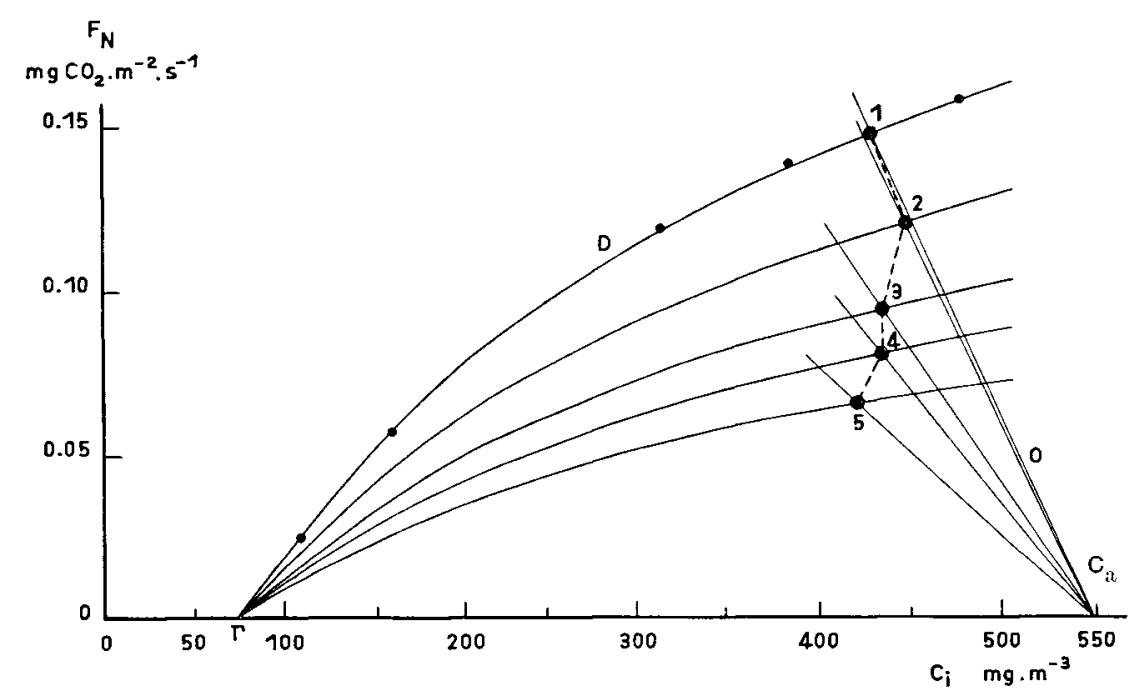

FIs; 2

Relation entre lintensité de photosynthèse nette $\left(F_{x}\right)$

et la concentration intercellulaire en $\mathrm{CO}_{2}\left(c_{i}\right)$ pour la provenance ECOUVES au 27 aồt 1984 (courbe I, figure 1$)$.

\section{Rate of net $\mathrm{CO}_{2}$ assimilation $\left(F_{\mathrm{S}}\right)$ against intercellular $\mathrm{CO}$, concentration $\left(c_{i}\right)$ at August 27th 1984 for the provenance Ecouves. (curve I, figure 1).}

Chaque point expérimental 1,2,3, 4 et 5 est l'intersection entre une fonction de demande (D) et une fonction d'offre (O) (voir texte). Les points expérimentaux correspondent aux valeurs suivantes de $\Delta \mathrm{c}^{\prime}: 3470$ (1), 5200 (2), 6940 (3), 8670 (4) et 11270 (5) $\mathrm{mg} \cdot \mathrm{m}^{-3}$.

Each experimental point 1,2,5,4 and 5 is the intersection of a demand function (D) and a supply function (O). Leaf-to-sir humidity difference, 3470 (point 1), 5200 (2), $6940(3), 8670$ (4) and $11270(5) \mathrm{mg} \cdot \mathrm{m}^{3} \cdot \mathrm{C}_{4}$, atmospheric $\mathrm{CO}_{2}$ concentration. $\Gamma, \mathrm{CO}_{2}$ compensation point of net assimilation.

Pour cela, il est nécessaire de représenter les points expérimentaux sous la forme d'un graphique $\left(\mathrm{F}_{x}, \mathrm{c}_{\mathrm{i}}\right), \mathrm{c}_{\mathrm{i}}$ étant déterminé pour chaque point suivant les équations (1) et (2). Une telle relation obtenue pour la provenance Ecouves au 
27 aont 1984 (situation de la courbe I de la figure 1) est représentéc par une ligne en tireté épais sur la figure 2. D'évidence, la trajectoire suivie par $F_{N}$ lors du passage du point 1 au point 5 ne se situc pas sur une courbe $D\left(c_{i}\right)$ unique (celle passant par le point 1). Cela signilie que les processus mésophylliens de la photosynthèse sont responsables, au moins en partie, de la diminution de $F_{x}$ lorsque $\Delta c^{\prime}$ augmente.

Chaque point expérimental de la figure 2 apparaît (JONES, 1973 ; RASCHKE, 1979) comme l'intersection d'une fonction de demande (D) qui représente l'efficacité des processus mésophylliens de la photosynthèse et d'une fonction d'offre (droite de pente- $g_{s}$ et passant par le point $(\mathrm{ca}, \mathrm{o})$ ) qui fixe $\mathrm{c}_{i}$ à travers le déterminisme de l'équation (1).

L'ensemble des fonctions $D$ de la figure 2 a été déduit de la courbe que nous avons établie expérimentalement pour les conditions du point 1, par une transformation telle que pour le passage d'une courbe $D_{1}$ à une courbe $D_{2,2}$, le rapport $D_{1}$ $\left(c_{i}\right) / D_{2}\left(c_{i}\right)$ soit indépendant de $c_{i}$. Ehleringer \& CoOK (1984) ont obtenu des variations de la fonction $D$ identiques dans le cadre d'une étude des effets de potentiels hydriques décroissants pour Encelia farinosa.

\section{TABLEAU 1}

Part stomatique dans la diminution de $F_{N}$ représentée sur la figure 2.

Pour le détail des calculs voir annexe 1.

Stomatal component of the decrease in $F_{N}$ shown in figure 2. For the details of calculations see appendix 1.

\begin{tabular}{|c|c|c|}
\hline Etape & $\begin{array}{l}\text { (a) Hypothèse } \\
\text { «stomates d'abord } \\
\text { (a) Hypothesis } \\
\text { \&stomata first» }\end{array}$ & $\begin{array}{l}\text { (b) Hypothèse } \\
\text { «mesophylle d'abord } \\
\text { (b) Hypothesis } \\
\text { «mesophyll first». }\end{array}$ \\
\hline $1-2$ & $4 \%$ & $2 \%$ \\
\hline $2-3$ & $27 \%$ & $15 \%$ \\
\hline $3-4$ & $18 \%$ & $14 \%$ \\
\hline $4-5$ & $30 \%$ & $20 \%$ \\
\hline
\end{tabular}

Pour chaque élape du passage du point 1 au point 5 de la figure 2 , il est possible de déterminer la composante stomatique et la composante mésophyllienne de la diminution de $F_{X}$ en faisant intervenir en premier soit une diminution d'origine stomatique, soit une diminution d'origine mésophyllienne (pour le détail des calculs, voir annexe 1). Les données du tableau 1 , relatives à l'exemple de la figure 2 , font apparaître que les processus mésophylliens expliquent en très grande partie les diminutions de $F_{\mathrm{N}}$. La part stomatique augmente lorsque $\Lambda \mathrm{c}^{\prime}$ augmente, mais ne dépasse, pour aucune des étapes considérées, 30 p. 100 de la diminution totale. Ce schéma s'applique à l'ensemble des situations que nous avons étudiées, pour les deux provenances. 


\subsection{Conclusions pour la détermination de $\partial \mathrm{F}_{\mathrm{N}} / \partial \mathrm{g}_{\mathrm{s}}$}

L'importance des processus non stomatiques dans la diminution de Fx que nous avons pu établir pour l'ensemble des courbes de la figure 1 interdit le recours à la méthode graphique pour l'estimation de $\partial \mathrm{F}_{\mathrm{N}} / \partial \mathrm{g}_{*}$ dans l'équation (7).

La représentation des données sous la forme illustrée par la ligure 2 permet une détermination de $\partial \mathrm{F}_{\mathrm{N}} / \partial \mathrm{g}_{\mathrm{s}}$ pour chacun des points expérimentaux, $\partial \mathrm{F}_{\mathrm{x}}$ étant la variation de $F_{X}$ engendrée par unc variation infinitésimale $\partial g_{s}$ pour une fonction $D$ bien déterminée. Lé calcul de $\partial F_{Y} / 3 g_{s}$ est exposé dans l'annexe 2 . On a déterminé $\partial F_{x} / \partial g_{s}$ pour l'ensemble des données relatives aux situations de la figure 1 et on a déduit les rapports $\partial T R / \partial F_{x}$ grâce à l'équation (7). Les variations de $\partial T R / \partial F_{x}$ en fonction de $\Lambda c^{\prime}$ sont montrées par la figure 3. Pour les deux périodes étudiées, lorsque la sécheresse atmosphérique augmente, on note une bonue stabilité de $\lambda$ pour la provenance Neßsıs, cependant que pour la provenance Ecouves $\lambda$ est à la fois plus instable et supérieur en valeur.


FIG. 3

Relations entre $\partial T R / \partial F_{x}$ et la différence de concentration an vaprar d'eau entre les fe'uilles et l'air $\left(\Delta c^{\prime}\right)$ pour les provernances Ecouves (@) et Nibias (O).

Relationships berwecen the $\mathrm{DTR} / \mathrm{\partial} F_{x}$ ratio and the leaf-to-air hamidity difference (AC) for the provenances ECOUVEs (-) and Nebias $(0)$.

(x) : valeurs obtenues pour la provenance Ecouves au 28 septembre 1984 en utilisant la méthode graphique directe pour l'estimation de $\partial \mathrm{F}_{\mathrm{x}} / \mathrm{\partial g}_{\mathrm{*}}$.

(x) : values obtained for Ecouvis at September 28 th 1984 using the slope of the $F_{x}\left(g_{N}\right)$ curve for the determination of $\partial F_{x} / \partial g_{s}$ and $\partial T R / \partial F_{x}$.

Pour les données du 28 septembre 1984 relatives à Ecouves, on a également déterminé $\partial F_{x} / \partial g_{s}$ en l'assimilant à la valeur de la pente de la relation entre $F_{x}$ et $g_{s}$. Les valeurs de $\mathrm{OTR} / \partial \mathrm{F}_{\mathrm{X}}$ áinsi obtenues (fig. 3) sont fondamentalement différentes des valeurs obtentes suivant la méthode que nous avons proposée. 


\section{Conclusions}

Une utilisation trop systématique de la théorie proposée par CownN (1977) ef Cowan \& Farouirar (1977) peut faire aboutir à des résultats et des conclusions fondamentalement erronées. Les risques d'erreur sont liés à l'appréciation du statut réel de la relation entre $F_{N}$ et $g_{s}$. L'utilisation de cette relation pour la détermination directe de $\partial F_{N} / \partial g_{s}$, puis $\partial T R / \partial F_{N}$ n'est permise que si les variations de $F_{K}$ sont entièrement expliquées par celles de $\mathrm{g}_{s}$.

La représentation des données sous la forme de relations $\left(F_{x}, c_{i}\right)$ et la décomposition des variations de $F_{\mathrm{Y}}$ en composantes stomatique et non stomatique proposée par Prioul et al. (1984) permettent d'une part d'établir le statut réel des relations $\left(F_{N}, g_{s}\right)$ et d'autre part de déterminer le terme $\partial T R / \partial F_{X}$ pal une méthode graphique simple, quelle que soit la nature précise de la relation entre $F_{x}$ et $g_{s}$.

L'utilisation de cette méthode suppose cependant que soit connue la variation de la fonction $\mathrm{D}$ au cours de la période étudiée. Cette information est assez aisément accessible pour les études de laboratoire où l'on fait varier un seul facteur expérimental à la fois mais l'extrapolation à des études faites en conditions naturelles paraît beaucoup plus délicate et reste tributaire de progrès à réaliser dans la modélisation du fonctionnement photosynthétique à l'échelle de la feuille.

L'ensemble des observations, essentiellement d'ordre méthodologique, quic nous avons pu formuler dans le présent article ne remettent nullement en question la validité de la théorie d'optimisation de Cowan (1977) et de Cowan \& Farounar (1977). Nous nous proposons de montrer l'intérêt des applications qui peuvent en être faites pour la caractérisation écophysiologique des espèces ou provenances d'arbres forestiers dans un prochain article qui sera consacré à une étude plus complète du fonctionnement hydrique des deux provenances ayant servi de support au présent travail.

\section{Reçu en décembre 1984.}

Accepté en mai 1985.

\section{Remerciements}

Je tiens à remercier MM. G. Aussenac et E. Dreyer pour l'intérêt qu'ils ont manifesté pour ce travail et les critiques constructives faites à propos du manuscrit.

\section{Surmmary}

Optimization of the relations between transpiration and plotosynthesis. Observations related to the methods of studies

Cowan and Farquiar (1977) have proposed an optimization theory for the relations between transpiration and photosynthesis in the higher plants. For a given lime interval, optimization of the water cost for net $\mathrm{CO}_{3}$ assimilation requires the instantaneous

$$
\frac{\partial T R / \partial g_{s}}{\partial F_{s} / \partial g_{s}}
$$


ratio to be constant with time (TR, transpiration rate: $\mathrm{F}_{\mathrm{x}}$, net $\mathrm{CO}_{2}$ assimilation rate; $\mathrm{g}_{*}$, stomatal conductance for $\mathrm{CO}_{2}$ diffusion).

Studies related to the optimization theory have experimentally often consisted in the cxamination in laboratory of changes in $F_{x}$ and $T R$ in response to increasing leaf-to-air humidity differences $\left(\Delta \mathrm{c}^{\prime}\right)$, and $\mathbf{F}_{x}$ changes were generally only attributed to changes in $\mathrm{g}_{*}$ Thus, $\partial F_{x} / \partial g_{s}$ would be determined directly as the slope of the $F_{x}\left(g_{s}\right)$ curves (fig. 1).

This direct method cannot be used for Abies alba, since for this species $F_{X}$ changes in response to increasing $\Delta \mathrm{c}^{\prime}$ values did not correspond to a constant demand function in a plot of $F_{X}$ against intercellular $\mathrm{CO}_{2}$ concentration (fig. 2), thus indicating a significant non-stomatal effect in the $F_{x}$ changes (appendix 1, table 1).

An alternative method is proposed here - based on an analysis of the datas in terms of demand and supply functions (fig. 2) - through which it is possible to determine the $\partial F_{s} / \partial g_{s}$ and

$$
\frac{\partial T R / \partial g_{s}}{\partial F_{N} / \partial g_{s}}
$$

ratios (appendix 2) whatever the nature of the relations between $F_{X}$ and $g_{s}$ may be.

\section{Annexe 1}

Détermination des composantes stomatique et mésophyllienne d'une variation de $\mathrm{F}_{\mathrm{X}}$ (d'après Prioul et al., 1984)

Sur la figure $A$ 1, on a représenté la décomposition d'une diminuticn de $F_{X}$ lors du passage d'un état 1 à un état voisin 2 .

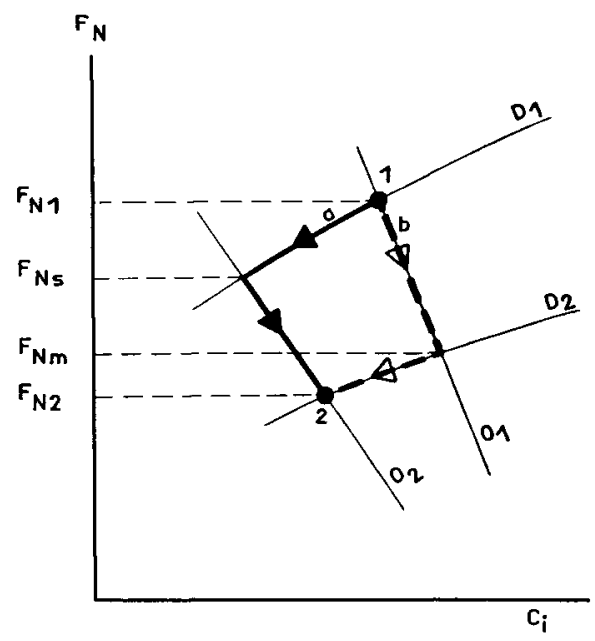

FIG. A 1

Décomposition d'une diminution de photosynthèse nette $\left(F_{x}\right)$

lors du passage de l'état 1 à l'état 2 suivant l'hypothèse «stomates d'abord》 (a) et suivant l'hypothèse «mésophylle d'abord》(b).

Decomposition of a change in the photosynthetic rate $\left(F_{x}\right)$ from 1 to 2 using hypothesis «stomata first》(a) and using hypothesis «mesophyll first》 (b). (D), demand functions; $(O)$, supply functions; $c_{i}$, intercellular $\mathrm{CO}_{2}$ concentration. 
a) si la diminution d'origine stomatique intervient en premier, la composante stomatique est, en pourcentage de la diminution totale :

$$
\frac{F_{N 1}-F_{N s}}{F_{N 1}-F_{N 2}} \cdot 100
$$

b) si la diminution d'origine mésophyllienne intervient en premier, la composante stomatique a pour valeur :

$$
\frac{F_{\mathrm{Nim}}-F_{\mathrm{N} 2}}{F_{\mathrm{N} 1}-F_{\mathrm{N} 2}} \cdot 100
$$

Annexe 2

Détermination graphique de $\partial \mathrm{F}_{\mathrm{N}} / \partial \mathrm{g}_{*}$ et de $\mathrm{\partial TR} / \partial \mathrm{F}_{\mathrm{N}}$ (lig. A 2)

La différenciation de l'équation (1) fournit :

$$
\mathrm{d} \quad \mathrm{F}_{\mathrm{N}}=\left(\mathrm{c}_{\mathrm{i}}-\mathrm{c}_{\mathrm{i}}\right) \mathrm{dg}_{\mathrm{s}}-\mathrm{g}_{\mathrm{s}} \mathrm{dc}_{\mathrm{i}}
$$

$\beta$ étant défini comme indiqué sur la figure A 2, on a pour une fonction de demande donnée :

$$
\partial \mathrm{F}_{\mathrm{N}}=\operatorname{tg} \beta \cdot \partial \mathrm{c}_{\mathrm{i}}
$$

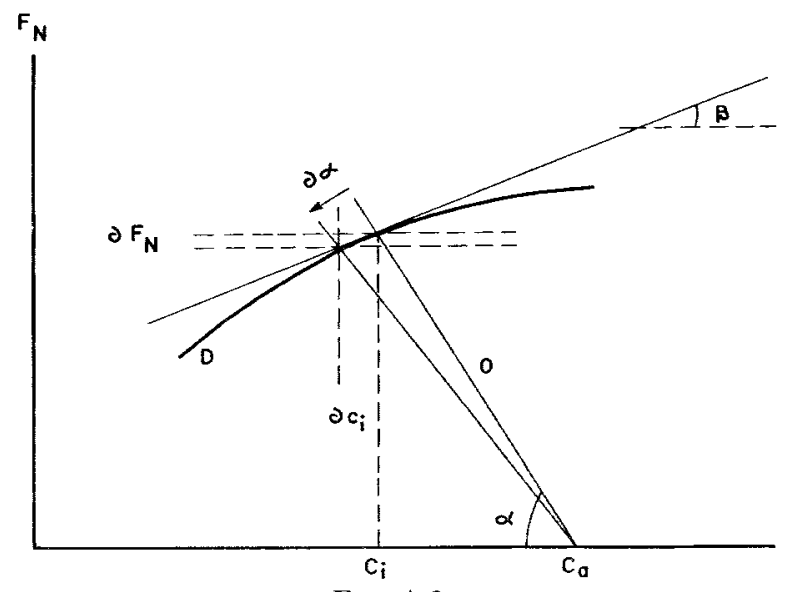

FIG. A 2

Détermination de la sensibilité de la photosynthèse nette $\left(F_{*}\right)$ à la conductance stomatique $\left(g_{s}=t_{g} \alpha\right)$

Determination of the $\partial F_{s} / \partial g_{s}$ ratio. $C_{i}$, intercellular $\mathrm{CO}_{2}$ concentration; $c_{t}$, atmospheric $\mathrm{CO}_{2}$ concentration. $g_{s}=t_{t} \alpha$. 
En combinant (9) et la forme différentielle partielle de (8) (fonction de demande constante), il vient :

$$
\frac{\partial F_{x}}{\partial g_{s}}=\left(c_{i t}-c_{i}\right) \frac{t_{x p} \beta}{t_{x} \beta+g_{s}}=\left(c_{i 1}-c_{i}\right) \frac{t_{x} \beta}{t_{s} \beta+t_{g} \alpha}
$$

Soit encore, si l'on fait remarquer que $t_{k} \beta$ peut être assimilé à une conductance mésophyllienne $\left(\mathrm{g}_{m}{ }^{*}\right)$ définie localement au voisinage de $\mathrm{c}_{\mathrm{i}}$ (Jonks \& SLATYER, 1972) :

$$
\frac{\partial F_{N}}{\partial g_{*}}=\left(c_{i 1}-c_{i}\right) \frac{g_{m}^{*}}{g_{m+1}^{*}+g_{*}}
$$

d'où, d'après l'équation (7) :

$$
\frac{\partial T_{1:}}{\partial F_{x}}=1.6 \frac{c^{\prime} f-c^{\prime} a}{c_{i 1}-c_{i}} \cdot \frac{g_{n}{ }^{*}+g_{s}}{g_{n}^{*} *}
$$

\section{Références bibliographiques}

Aussenac G., 1980. Comportement hydrique de rameaux excisés de quelques espèces de sapins et de pins noirs en phase de dessication. Ann. Sci. For.. 37 (3), 201-215.

Aussfinac G., Granter A., 1978. Quelques résultats de cinétique journalière du potentiel de sève chez les arbres foresticrs. Ann. Sci. For., 35 (1), 19-32.

Bethenod O, Bousquet J.F., Lafiray D., Louguet P., 1982. Réexamen des modalités d'action de l'ochracine sur la conductance stomatique des feuilles de plantules de blé, triticum aestivum L., CV «Etoile de Choisy». Agronomie, 2 (1), 99-102.

Cornic G., Prioul J.L., Lounsson G., 1983. Stomatal and non stomatal contribution in the decline in leaf nct $\mathrm{CO}_{2}$ uptake during rapid water stress. Physiol. Plant., 58, 195-301.

Cowan I.R., 1977. Stomatal behaviour and environment. Adv. Bot. Res., 4, 117-228.

COWAN I.R., 1982. Regulation of water use in relation to carbon gain in higher plants. In : Lange O.L., Nobez P.S., Osmond C.B., Ziegler H. (eds). Fncyclopedia of plant physiology, new series, vol. $12 \mathrm{~B}$, Physiololocal plant Ecology II, Springer-Verlag, Berlin, Heidelberg, New York, 590-613.

Cowan I.R., Farquhar G.D., 1977. Stomatal function in relation to leal metabolism and environment. Symp. Soc. Exp. Biol., 31, 471-505.

Farquiar G.D., 1978. Feedforward responses of stomata to humidity. Aust. J. Plami Physiol., 5, 787-800.

Farquhar G.D. Schulze E.D., Küppers M., 1980 a. Responses to humidity by stomata of Nicotinia glauca L. and Corylus avellana L. are consistent with the optimization of Carbon dioxide uptake with respect to water loss. A ust. J. Plant. Physiol., 7, 315-327.

Farquhar G.D., Cafmmerer S., Berry J.A., 1980 b. A biochemical model of photosynthetic $\mathrm{CO}_{2}$ assimilation in leaves of $C_{: 3}$ Species. Planta, 149, 78-90.

Hall A.E., Schulze E.D., 1980. Stomatal response to environment and a possible interrelation between stomatal effects on transpiration and $\mathrm{CO}_{.}$assimilation. Plant Cell Environ., 3, 467-474. 
Jones H.G., 1973. Limiting factors in photosynthesis. New Phytol., 72, 1089-1094.

JONES H.G., 1980. Interaction and integration of adaptative responses to water stress : the implications of an unpredictable environment. In : TURNER N.G., KrAmer P.J. (eds). Adaptation of plants to water and high temperature stress. Wiley-Interscience, New York, 353-365.

JoNES H.G., St.ATYER R.O., 1972. Effects of intercellular resistances on estimates of the intracellular resistance to $\mathrm{CO}_{\text {. }}$ uptake by plant leaves. Aust. J. Biol. Sci., 25, 443-453.

Meinzer F.C., 1982. The effect of vapor pressure on stomatal control of Gas exchange in Douglas fir (Pseudotsuga menziesii) saplings. Oecologia (Berl.), 54, 236-242.

Prioul J.L., Cornic G., Jones H.G., 1984. Discussion of stomatal and non stomatal components in leaf photosynthesis decline under stress conditions. In : SYBESMA G. (ed.), Advances in Photosynthesis Research, Vol. IV, Martinus Nijhoff/ Dr. W. Junk publishers, The Hague, Boston, Lancaster, 375-378.

RASCHKE K., 1975. How stomata resolve the dikemna of opposing priorities. Phil. Trans. R. Soc. Lond. B., 273, 551-560.

RaschKe K., 1979. Movements of stomata. In : Haupr W., Feinleib M.E. (eds), Physiology of movements. Encyclopedia of plant physiology new series, vol. VII, Springer, Berlin, Heidelberg, New York, 383-441.

Wong S.C., Cowan I.R., Farquhar G.D., 1979. Stomatal conductance corrclates with photosynthetic capacity. Nature, 282, 424-426. 